\title{
El impacto de las aplicaciones móviles en la gestión empresarial en Latinoamérica
}

\section{The impact of mobile applications in business management in Latin America}

Msc. Jorge Misael Merchán Riera

Mae. Christian Josuepht Moreno Rodríguez

Mae. Melvin Leonardo López Franco

Mae. Joffre Arturo Santamaría Yagual

Universidad de Guayaquil, Ecuador

Autor para correspondencia: jorge.merchanr@ug.edu.ec, christian.morenorod@ug.edu.ec, melvin.lopezf@ug.edu.ec, joffre.santamariay@ug.edu.ec.

Fecha de recepción: 23 de Noviembre de 2016 - Fecha de aceptación: 10 de Enero de 2017

\section{Resumen}

El presente estudio tiene como finalidad explorar, documentar y posteriormente analizar las repercusiones que están teniendo diversas aplicaciones de software móvil en la gestión de las empresas en Latinoamérica. Es menester tomar en cuenta el impacto de las tecnologías de la información y comunicación (TIC) en el área de la administración de empresas para así lograr un aprovechamiento considerable de los nuevos avances en tecnología de la comunicación que podrían significar una mejora en los procesos gerenciales de nuestras organizaciones a nivel regional, incluso con la posibilidad de impulsarlas a un nivel de competitividad internacional. En esta investigación dejamos claro el impacto que estos cambios vertiginosos están causando en América Latina y proponemos alternativas para hacer un correcto uso de ellas.

Palabras clave: Aplicaciones móviles; gerencia empresarial; América Latina

\begin{abstract}
The purpose of the following paper is to explore, document and subsequently analyze the repercussions of various mobile software applications in the management of companies in Latin America. It is necessary to take into account the impact of information and communication technologies (ICT) in the area of business administration in order to achieve a considerable use of new advances in technologies of communication that could signify an improvement in the management processes of our organizations at regional levels, even with the possibility of promoting them to a level of international competitiveness. In this research we make clear the impact that these vertiginous changes are causing in Latin America and we propose alternatives to make a correct use of them.
\end{abstract}

Key words: Mobile apps; business management; Latin America 


\section{Introducción}

Esta investigación tiene como objetivo explicar de manera concisa los efectos que tienen las aplicaciones móviles en el área de la gestión empresarial (también conocida como administración de empresas) en América Latina, se puede evidenciar que el impacto de las tecnologías de la información y la comunicación (conocidas como "TIC") no se limita al sector para el que se producen, sino que a su vez se extiende a todos los sectores de producción y consumo en la sociedad.

Esto también es válido para la telefonía móvil y su área más específica de las aplicaciones móviles. Además, la influencia de dichas aplicaciones aumenta a medida que los efectos de la red lo hacen; Es decir, cuando aumenta el número de personas que las utilizan en sus rutinas diarias.

Por otra parte, es un área que muestra una clara mejoría con el tiempo - los dispositivos móviles incorporan más y mejores aplicaciones, mientras que la calidad de las comunicaciones también se desarrolla. La cobertura de infraestructuras y servicios crece y, al mismo tiempo, los precios muestran una clara tendencia a la baja en la mayoría de los países de la región.

Por último, las aplicaciones móviles también generan innovación, ya que promueven y facilitan la invención y la producción de nuevos servicios, productos o procesos en el ámbito gerencial. Los ejemplos son comunes, desde el uso de "Skype" para concertar una reunión con tus subordinados, hasta la banca móvil para cancelar sueldos y salarios, tendencias que están modificando la gerencia tanto en áreas rurales como urbanas de Latinoamérica.

A raíz de todo lo antes mencionado, es importante entender el impacto que estos cambios han generado en la gerencia de nuestras sociedades latinoamericanas, comprendiendo que los beneficios asociados a la difusión de una tecnología de uso general van más allá de su aplicación a los procesos de negocio y permiten generar mejoras en la calidad y eficiencia de los procesos gerenciales dentro de las organizaciones.

Al igual que con los teléfonos fijos, la difusión de la telefonía móvil y sus nuevas aplicaciones digitales implica cambios en la organización cotidiana de la vida privada, lo que a su vez repercute en las actividades laborales tomando en cuenta que ninguna organización funciona como un ente aislado de su contexto.

Ya se trate de empresas grandes o pequeñas, de empresas formales o informales, desde un punto de vista puramente económico, podemos identificar una serie de áreas en las que la presencia de aplicaciones móviles está impulsando cambios. Ya sea que funcione o no en combinación con la telefonía fija, la comunicación inalámbrica e instantánea permite una mayor flexibilidad de gestión y acelera los procesos que dependen de esta dentro de las organizaciones, uno de ellos siendo el gerencial.

\section{Marco Teórico}


Según The Computer Language Company Inc. (2016) PC Magazine, las aplicaciones móviles pueden definirse Como: "Es una aplicación de software que se ejecuta en un teléfono inteligente, tableta u otro dispositivo portátil. En contraste con las aplicaciones de escritorio. Se dividen en: aplicación móvil nativa, versión móvil y tienda de aplicaciones en línea”.

Según Idalberto Chiavenato (2004) "Introducción a la Teoría General de la Administración”, Séptima Edición, McGraw-Hill Interamericana, Pág. 10. La gestión empresarial, también conocida como administración de empresas puede definirse como: "el proceso de planificar, organizar, dirigir, y controlar el uso de los recursos para lograr los objetivos organizacionales".

De la misma manera para los autores Robbins y Coulter (2004) “Administración”, Octava edición. La gestión empresarial o administración de empresas es considerado como: "la coordinación de las actividades de trabajo de modo que se realicen de manera eficiente y eficaz con otras personas a través de ellas”.

Daremos una última definición de gestión empresarial para dejar en claro los procesos específicos que esta contempla, para esto usaremos la definición de los autores Díez de Castro, García del Junco, Martín Jiménez y Periáñez Cristóbal (2001) “Administración y Dirección”, McGraw-Hill, Madrid. La cual conceptualiza a la gestión empresarial como: "el conjunto de las funciones o procesos básicos (planificar, organizar, dirigir, coordinar y controlar) que, realizados convenientemente, repercuten de forma positiva en la eficacia y eficiencia de la actividad realizada en la organización".

\section{Marco Metodológico}

Para esta investigación usaremos un método documental exploratorio, considerando la naturaleza de un estudio a nivel de América Latina. Para esto se revisaron diversas fuentes disponibles sobre estudios relacionados con el crecimiento en el uso de aplicaciones móviles en nuestra región y de cómo este crecimiento podría impactar en las formas y maneras de la gestión empresarial tradicional.

Algunas de las investigaciones usadas como referencia para la presente investigación fueron: Mobile Marketing Association (2015) Latín América Is Fastest-Growing Mobile Ad Market. Una investigación que se realizó para determinar el crecimiento del mercado de publicidad móvil en Latinoamérica, dicha investigación contiene datos que aportan substancia y base a algunas de las afirmaciones hechas en el presente estudio.

También fueron tomados en consideración diversos estudios de telefónicas a nivel mundial que aportaban datos sobre la región de América Latina y del incremento al acceso de telefonía móvil y de cómo este estaba directamente ligado a un mayor uso de aplicaciones móviles, en especial la investigación de Vodafone (2013) Impact of Mobile Technologies On Enterprise: Strategies, Success, Factors, Recommendations.

De igual forma nos referimos al International Journal of Engineering \& Technology IjetIjens Vol: 10 No: 06 (2010) Mobile Application and Its Global Impact. Investigación que se 
encargó de realizar un sondeo a nivel global sobre el impacto que habían tenido hasta la fecha las aplicaciones móviles en el área empresarial, tratando dentro de ello el tema de la gestión o administración de estas empresas.

Para finalizar, también se utilizaron datos tomados de fuentes documentales como revistas especializadas en el tema de las aplicaciones móviles y tecnología empresarial: La revista Business Insider con su investigación titulada Latín América has a tremendous app opportunity in front of it (2016), nos proporcionó datos importantes para el desarrollo de las conclusiones, el medio de comunicación RCR Wireless News con su artículo LatAm: The future of Mobile apps (2015) el cual describe las oportunidades de crecimiento para el mercado de las aplicaciones móviles en nuestra región. Ambas fuentes fueron tomadas para desarrollar un hilo argumentativo a partir de las afirmaciones de sus autores expertos en el área de las aplicaciones móviles.

Las técnicas investigativas utilizadas en el presente estudio son:

$\checkmark$ Documentación de fuentes secundarias y terciarias

$\checkmark$ Análisis de diversos estudios globales y regionales referentes al tema.

$\checkmark$ Graficación de la data más relevante para el presente estudio.

$\checkmark$ Desarrollo de una conclusión en base a los datos obtenidos.

\section{Análisis}

Con los negocios digitales difuminando la línea entre el mundo físico y el mundo digital, las aplicaciones móviles centradas en el consumidor están desempeñando un papel importante en América Latina, según datos de la empresa de telecomunicaciones Vodafone 7 (2013) la mayoría de las aplicaciones móviles sincronizarán, recopilarán y analizarán datos personales sobre los usuarios y de sus preferencias, aún con toda esta evidencia a favor de tomar en consideración a las aplicaciones móviles como herramientas importantes en el área administrativa la mayoría de los gerentes y administradores no están considerando el impacto profundo que tienen las aplicaciones móviles en su estructura gerencial.

La Mobile Marketing Association 4 (2015) predice que, para 2017, los dispositivos portátiles manejarán el 50 por ciento de las interacciones totales del mercado de las aplicaciones en Latinoamérica. Hasta la fecha, la mayoría de las aplicaciones se han desarrollado para soportar interacciones específicas entre empresas y consumidores. Por ejemplo, algunos usan los datos de ubicación para ofrecer información contextualmente relevante, y algunos también recogen otra información sobre sus usuarios -como género y grupo de edad- para refinar aún más la interacción.

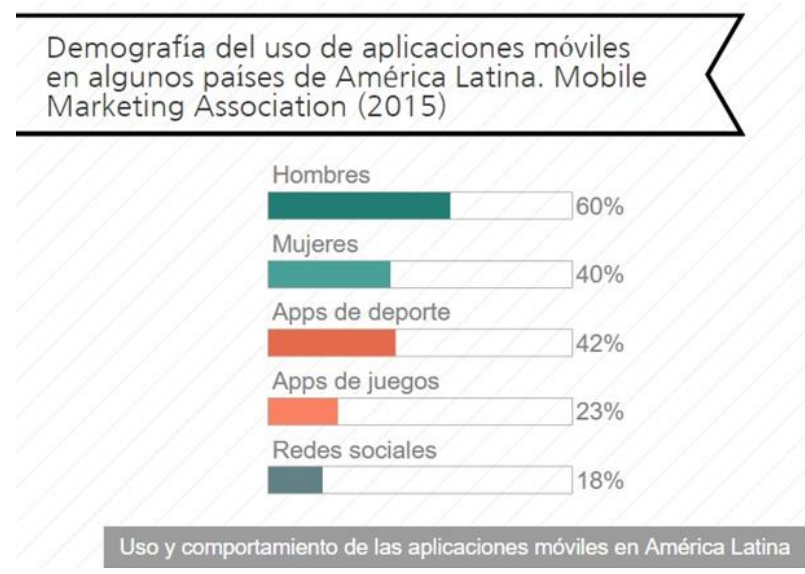

Según datos del International Journal of Engineering \& Technology 3 (2010) los datos personales a menudo se recogen únicamente en apoyo de los requisitos de una aplicación móvil y no se consideran un activo dentro de la infraestructura de información general de una organización, y menos aun cuando a lo que a procesos gerenciales concierne. En consecuencia, aunque se accede a estos datos y se almacena potencialmente en apoyo de una aplicación, no se gestiona como dichos datos pudieran beneficiar en el accionar administrativo.



También es importante mencionar que la línea entre el uso aceptable e inaceptable de los datos del consumidor de las aplicaciones móviles puede ser muy delgada, y se vuelve aún más delgada a medida que los datos recogidos se vuelven más detallados y personales. Por ejemplo, las organizaciones que recopilan datos biométricos a través de aplicaciones móviles vinculadas a dispositivos portátiles podrían verse tentadas a monetizar estos datos revendiéndolos. Los 
gerentes deben ser los garantes de la honestidad y la transparencia al momento de considerar el uso de los datos de sus subordinados obtenidos a través de aplicaciones móviles.

Vodafone 7 (2013) nos da un caso interesante de esto en Brasil, dónde se demuestra que incluso en el caso de que los datos personales o biométricos sean anónimos, igual podría tener un impacto importante en la capacidad de una persona para obtener un seguro de salud adecuado si son identificados como pertenecientes a una categoría de riesgo por parte del personal gerencial, encargado a su vez del monitoreo de la data recogida en estas aplicaciones móviles de última generación.

Además, las aplicaciones móviles que utilizan terceras partes para la autenticación proporcionan datos sobre el comportamiento del personal, lo que ayudaría al mismo tiempo en las investigaciones de clima o cultural laboral.

Estos riesgos relacionados con los datos recopilados en aplicaciones móviles requieren que las organizaciones replanteen sus políticas gerenciales y ajusten su infraestructura de información para que esta cantidad importante de data personal de sus trabajadores sea empleada en aras del mejoramiento organizacional.

\section{Conclusión}

Luego de haber analizado concisamente el impacto que tienen las aplicaciones móviles en Latinoamérica es necesario hacer algunas recomendaciones para el área gerencial de las empresas a nivel de nuestra región, es menester aprovechar el crecimiento del mercado para aplicar estrategias novedosas que coloquen a nuestras organizaciones a niveles altos de competitividad, no sólo en los mercados nacionales o regionales, sino también a nivel internacional aprovechando el "boom" de las aplicaciones móviles y su sinfín de utilidades, es el deber del gerente estudiar el contexto de su respectiva empresa y posterior a un análisis determinar qué aplicaciones se adaptarían mejor a su realidad organizacional y de qué manera podrían usarse para el beneficio de todos. Un par de sugerencias puntuales podrían ser:

\section{- Administrar la persistencia y la perecedera de los datos recopilados de las aplicaciones móviles}

Supervisar el acceso y el control de/a estos datos. Es importante asegurarse de que los datos personales recopilados de las aplicaciones móviles permanezcan privados, y que estén protegidos, anónimos y únicamente se acceda de acuerdo con las políticas de la organización. Una gestión adecuada de los acuerdos entre empleado y gerente es un aspecto importante de esto.

\section{- Controlar el uso compartido y la reutilización de datos de aplicaciones para móviles para otros fines}

Los datos de las aplicaciones para móviles, ya sean desplegados en las instalaciones o en la nube de cada empresa, no se administran actualmente como parte de la infraestructura de información de una organización, y los datos recopilados de las aplicaciones para móviles a menudo ni siquiera se almacenan. 
Las organizaciones deberían planear gestionar la información a través de implementaciones en la nube y en almacenamientos locales, ya que la combinación de todos los datos en las instalaciones o en un solo repositorio ya no es viable hoy en día.

Es importante entender los acuerdos de nivel de servicio para varios casos de uso (cada aplicación tiene acuerdos de uso - conocidos como SLA- diferentes con los cuales el gerente debe familiarizarse) que tienen acceso a datos de aplicaciones móviles y adaptar la capacidad de información de la empresa en consecuencia.

Por ejemplo, la colocación de datos de aplicaciones móviles con otros datos de aplicación dentro de las instalaciones locales puede ser una mejor opción para casos de uso, como el análisis offline y de red interna. Sin embargo, el uso de la virtualización de datos para combinar datos en la nube con datos en las instalaciones locales puede ser una mejor opción si la colocación no se ajusta a la administración o los SLA de un caso de uso. El uso de capacidades de integración de plataforma como servicio para la integración de servicios en la nube puede complementar las estrategias de integración de datos existentes moviendo datos de la nube a las instalaciones o de nube a nube según sea necesario.

Para finalizar, es conveniente mencionar algunas aplicaciones móviles que han sido usadas con éxito en el área de la gerencia empresarial, y de cómo estas podrían beneficiar a las empresas de la región de adaptarse su uso a las necesidades de América Latina:

1.- VMZINC: permite a los clientes ver productos desde cualquier lugar.

Un proveedor global de productos laminados de zinc, VMZINC ha lanzado recientemente una aplicación móvil que permite a los clientes navegar por los productos y filtrar/clasificarlos por sus características deseadas. La aplicación incluso permite a los usuarios buscar proyectos VMZINC cercanos y, a continuación, guía al usuario a la ubicación del proyecto. Como bien se ha mencionado antes, esta aplicación móvil permite mostrar sus productos a sus clientes en cualquier momento, dondequiera que se encuentren. En esencia, ayuda a sus clientes hacer negocios con ellos. Sus clientes pueden navegar y comprar sus productos o servicios desde cualquier lugar, en cualquier dispositivo. Es una aplicación con muchos usos en el área gerencial, un ejemplo de su uso es permitir a los gerentes la observación en tiempo real de la valoración de los productos o servicios de su empresa en tiempo real, ayudándole así en la toma de decisiones.

2.- Amplify Entertainment: ofrece una mejor manera de comunicarse.

Una compañía de DJ de bodas con sede en Florida, Amplify Entertainment utiliza aplicaciones móviles para conectarse en tiempo real con sus invitados. En lugar de gritar las peticiones, los invitados a la boda ahora pueden hacer solicitudes de canciones desde su mesa o la pista de baile utilizando sus teléfonos móviles. En el área gerencial, su uso pudiese destinarse a la comunicación en tiempo real entre gerente y demás miembros de la empresa para agilizar la resolución de problemas: Por ejemplo, en una empresa de transporte de cargas pesadas, el chofer puede comunicarle al gerente mediante esta aplicación que el camión necesita mantenimiento con urgencia. 
3.- Midland Medical: optimiza su proceso de recepción.

Un distribuidor de suministros médicos con sede en Nebraska, Midland Medical automatizó su proceso de recepción de inventario utilizando aplicaciones móviles. En el pasado, los empleados registraban los nuevos inventarios en papel y, a continuación, introducían manualmente los datos en su sistema. Ahora, utilizando teléfonos inteligentes y escáneres bluetooth, combinados con una aplicación móvil personalizada, los empleados escanearán instantáneamente nuevos inventarios directamente a su base de datos.

En el área gerencial son evidentes los beneficios que podría significar el uso de una aplicación para escaneo de documentos, reducir la burocracia y simplificar los procesos significaría un incremento en la eficiencia y eficacia de la empresa.

\section{Bibliografia}

BI Intelligence (2016) Latin America has a tremendous app opportunity in front of it, Business Insider.

Díez de Castro, García del Junco, Martín Jiménez y Periáñez Cristóbal (2001) “Administración y Dirección”, McGraw-Hill, Madrid.

Idalberto Chiavenato (2004) “Introducción a la Teoría General de la Administración”, Séptima Edición, McGraw-Hill Interamericana, Pág. 10.

International Journal of Engineering \& Technology IJET-IJENS Vol: 10 No: 06 (2010) Mobile Application and Its Global Impact.

Mobile Marketing Association (2015) Latin America Is Fastest-Growing Mobile Ad Market.

Robbins y Coulter (2004) “Administración”, Octava edición.

Roberta Prescott (2015) Latam: The future of mobile apps, RCR Wireless News.

The Computer Language Company Inc. (2016) PC Magazine.

Vodafone (2013) Impact of Mobile Technologies on Enterprises: Strategies, Success, Factors, Recommendations. 